\title{
The Effect of an Intramembrane Light-Actuator on the Dynamics of Phospholipids in Model Membranes and Intact Cells
}

\author{
Giuseppe M. Paternò,* Gaia Bondelli, Victoria Garcia Sakai, Valentina Sesti, Chiara Bertarelli, \\ and Guglielmo Lanzani*
}

Cite This: Langmuir 2020, 36, 11517-11527

Read Online

ACCESS | Llll Metrics \& More | 国 Article Recommendations | st Supporting Information

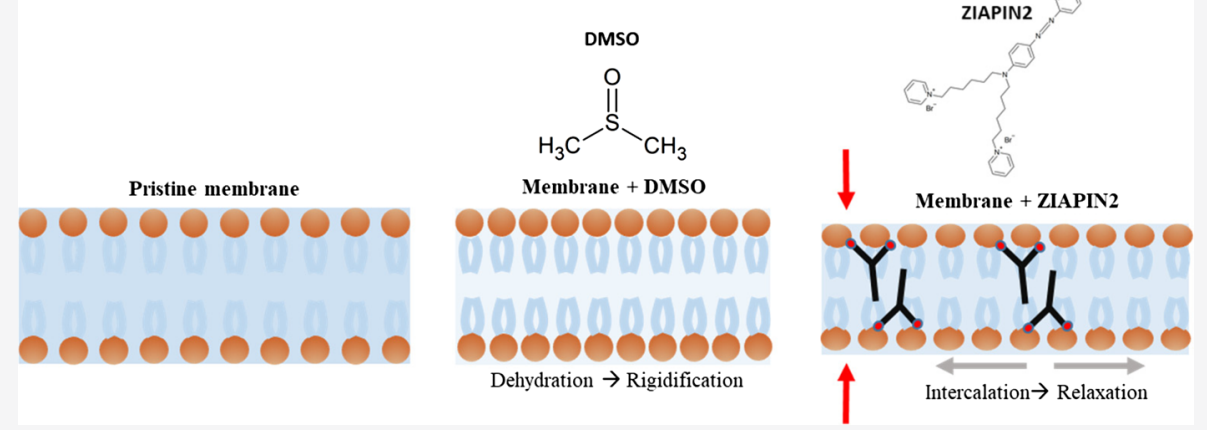

ABSTRACT: The noncovalent intercalation of amphiphilic molecules in the lipid membrane can be exploited to modulate efficiently the physical status of the membrane. Such effects are largely employed in a range of applications, spanning from drugdelivery to therapeutics. In this context, we have very recently developed an intramembrane photo-actuator consisting of an amphiphilic azobenzene molecule, namely ZIAPIN2. The selective photo-isomerization occurring in the lipid bilayer induces a photo-triggered change in the membrane thickness and capacitance, eventually permitting to evoke light-induced neuronal firing both in vitro and in vivo. Here, we present a study on the dynamical perturbation in the lipid membrane caused by ZIAPIN2 and its vehicle solvent, dimethyl sulfoxide. Effects on the dynamics occurring in the picosecond time range and at the molecular level are probed using quasi-elastic neutron scattering. By coupling experiments carried out both on model membranes and intact cells, we found that DMSO leads to a general retardation of the dynamics within a more dynamically ordered landscape, a result that we attribute to the dehydration at the interface. On the other hand, ZIAPIN2 partitioning produces a general softening of the bilayer owing to its interaction with the lipids. These data are in agreement with our recent studies, which indicate that the efficacy of ZIAPIN2 in triggering cellular signalling stems from its ability to mechanically perturb the bilayer as a whole, by forming lightsensitive membrane spanning dimers.

\section{INTRODUCTION}

The ability of cationic amphiphiles to intercalate into the lipid membrane of eukaryotic and prokariotic cells has been largely exploited to modulate membrane physical properties, with the final goal of providing new pharmacological platforms for therapeutics, i.e., for the development of membrane-targeted antibiotic and antiviral drugs. ${ }^{1-4}$ Cell membranes are complex two-dimensional fluids, whose dynamics can influence drug encapsulation and, vice versa, can be modified upon insertion of a guest molecule. Therefore, a detailed knowledge of the motions of phospholipids taking place at the molecular level is crucial for pharmacology, i.e., to evaluate possible effects on the integrity of the membrane and to elucidate the action mechanism. $^{5-7}$ Neutron scattering techniques, in particular, can be utilized effectively to investigate soft condensed matter, ${ }^{8}$ owing to the abundance of hydrogen nuclei that strongly interact with neutrons. ${ }^{9}$ Among these techniques, quasi-elastic neutron scattering (QENS) is well-suited to study molecular dynamics with timescales of $\approx 10^{-13}-10^{-9} \mathrm{~s}$ at length scales in the range of $1-30 \AA{ }^{10,11}$ thus allowing to map out a wide range of motions, from fast vibrations to slower diffusions, at the level of intramolecular and intermolecular distances. ${ }^{12}$ For these reasons, QENS has been used to probe the complex molecular motion of phospholipids and surfactants in supramolecular assembles and lipid bilayers, ${ }^{13-15}$ as well as in highly

Received: June 23, 2020

Revised: September 9, 2020

Published: September 9, 2020 
complex biological systems such as living cells. ${ }^{16,17}$ More specifically, QENS has also been exploited to investigate the effects of guest molecules of pharmaceutical interests on the structure and dynamics of the lipid membrane. ${ }^{18-22}$

We have recently developed an amphiphilic azobenzene molecule named ZIAPIN2, which partitions stably into the plasma membrane where it can work selectively as an efficient opto-mechanical actuator. ${ }^{23,24}$ In particular, while the darkadapted trans isomer causes a thinning of the lipid bilayer via a dimerization mechanism, illumination with visible light leads to a membrane relaxation that follows disruption of the azobenzene dimers. This, in turn, enables the cell membrane capacitance to be modulated and remarkably permits to evoke light-induced neuronal firing in vitro and in vivo. The chemical structure of ZIAPIN2 is engineered to be driven inside the plasma membrane and is characterized by a hydrophobic backbone containing a 4-4'diaminoazobenzene group substituted with an azepane ring on one side, two alkyl chains with six carbon atoms on the other side, and a hydrophilic head, namely, pyridinium bromides (Figure 1a). Molecular dynamics simulations have shown that ZIAPIN2 enters the lipid membrane very rapidly $(\approx 40 \mathrm{~ns})$ with virtually no energetic barrier to overcome. ${ }^{23}$

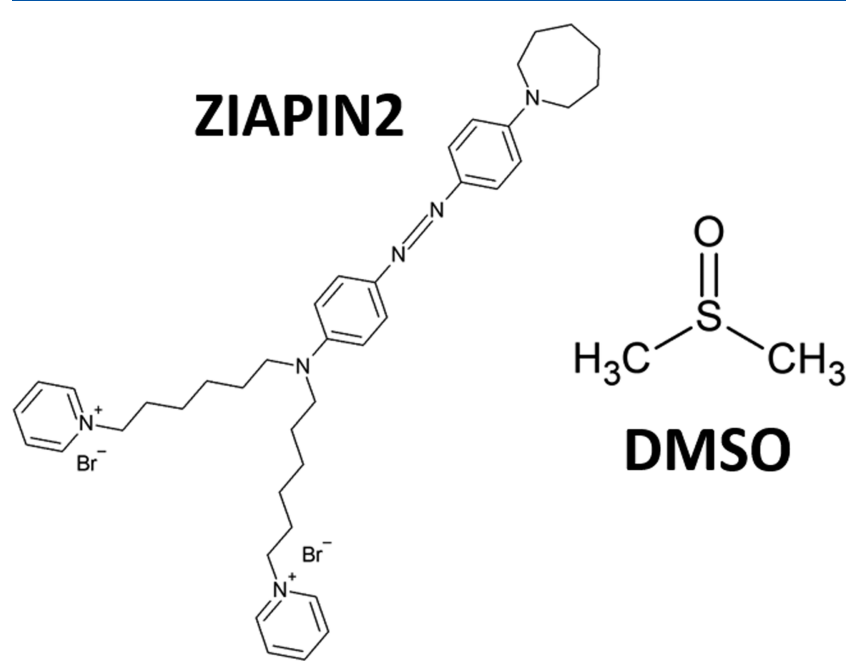

Figure 1. (a) Molecular structure of ZIAPIN2 and its vehicle solvent dimethyl sulfoxide (DMSO).

In this work, we employed QENS to study how ZIAPIN2 perturbs phospholipid dynamics in the picosecond time range at the molecular level (molecular distances of around 4 to 20 $\AA$ ). Control experiments with its vehicle solvent dimethyl sulfoxide (DMSO) were also carried out. Starting from a model membrane based on 1-palmitoyl-2-oleoyl-sn-glycero-3phosphocholine, POPC, our data suggest that while the addition of DMSO leads to a general retardation and restriction of the lipid dynamics, a result that we attribute to the dehydration at the water interface, ZIAPIN2 partitioning in the cell membrane causes a restoration of the POPC dynamical features, most likely thanks to its strong interaction with both the lipid head and acylic tails. Finally, we compared these findings with data acquired in living eukariotic cells (HEK293). Although the complex dynamical scenario in such samples hindered a direct quantitative/qualitative comparison with model membranes, interestingly, we could extract useful information on the dynamical trend of the in vitro and in vivo data upon addition of ZIAPIN2 and DMSO.

\section{EXPERIMENTAL SECTION}

ZIAPIN2 Synthesis. ZIAPIN2 was synthesized by reduction of the commercially available Disperse Orange 3 followed by the substitution of the amines with an excess of 1,6-dibromohexane. Full details about the synthesis can be found in our previous papers. $^{23,24}$

Liposome Preparation. POPC was purchased from Avanti Polar Lipids and used without further purification. Liposomes were prepared by extrusion following the experimental protocol suggested by the supplier. Briefly, a stock solution of lipids in chloroform/ methanol (1:1) was dried out under high vacuum via rotary evaporation $\left(40^{\circ} \mathrm{C}, 150 \mathrm{rpm}\right)$, and the resulting lipid film was kept overnight at $-20{ }^{\circ} \mathrm{C}$. The film was then hydrated with a buffer solution (10 mM Tris, $100 \mathrm{mM} \mathrm{NaCl}, \mathrm{pH} 7.4)$ and subjected to freeze-thaw cycles by alternately placing the flask in liquid nitrogen and in a water bath $\left(60^{\circ} \mathrm{C}\right)$. The lipid dispersion was then extruded at least 11 times through a polycarbonate membrane with a pore size of $100 \mathrm{~nm}$ to yield small unilamellar vesicles. Finally, the obtained dispersion was diluted to a concentration of $25 \mathrm{mg} / \mathrm{mL}$ for QENS characterization and kept at $4{ }^{\circ} \mathrm{C}$. ZIAPIN2 intercalation was performed by simply adding the molecule solution $(25 \mu \mathrm{M}$ in DMSO) to the liposome suspension followed by a vortexing step. The final concentration of DMSO in the POPC (HEK cells)/DMSO and POPC (HEK cells) /Ziapin 2 samples is the same, $1.25 \%$ in volume (molar fraction $=0.3 \%)$.

Cell Cultures. The Dulbecco's Modified Eagle Medium high glucose (DMEM-HG) culture medium, penicillin-streptomycin, PBS (phosphate buffered saline) tabs, trypsin-EDTA, L-glutamine, and deuterium oxide ( 99.9 atom \% D) were purchased from SigmaAldrich (UK). FBS (fetal bovine serum) was obtained from Gibco (UK). All procedures were performed using the immortalized Human Embryonic Kidney cells (HEK293) (Human Embryonic Kidney), purchased from ATCC. HEK293 cells are widely used in biotechnology because of their reliable and simple growth and propensity for transfection. Specifically, we have already employed HEK293 cells in our biophysical experiments to validate the optomechanical phenomenon brought about by ZIAPIN2 in a nonexcitable cell line that has an intrinsically low concentration of ion channels, to single out the effect on the plasma membrane itself. $^{23,24}$ Cells were tested for mycoplasma contamination with MycoFluor Mycoplasma Detection Kit (M-7006). HEK293 cells were cultured on-site (at the Life Science Laboratories of the Research Complex at Harwell) in cell culture flasks containing DMEM-HG supplemented with $10 \%$ heat inactivated FBS, $2 \%$ penicillin/ streptomycin, and $1 \% \mathrm{~L}$-glutamine. Culture flasks were maintained in a humidified incubator at $37{ }^{\circ} \mathrm{C}$ with $5 \% \mathrm{CO}_{2}$. Once at confluence, cells were enzymatically dispersed using a $1 \mathrm{x}$ trypsin-EDTA solution and left to grow for $24 \mathrm{~h}$ until $80 \%$ confluence prior to ZIAPIN2/ DMSO administration (from a $2 \mathrm{mM}$ solution in DMSO) at $25 \mu \mathrm{M}$ concentration followed by incubation for $7 \mathrm{~min}$ at $37{ }^{\circ} \mathrm{C}$, in a humidified $5 \% \mathrm{CO}_{2}$ atmosphere. After incubation, cells were washed with deuterated PBS. The cell pellet $\left(100 \mathrm{mg} / 1 \mathrm{~cm}^{3}\right.$, ca. $5 \times 10^{8}$ cells per sample) was prepared by cell harvesting (through trypsinization) followed by repeated (2x) PBS washing and centrifugation (at 1200 $\mathrm{rpm}$ for $5 \mathrm{~min}$ ) following the procedure described in a previous literature study. ${ }^{25,26} \mathrm{PBS}$ was used as an isotonic medium in order to avoid water exchange from the inside to the outside of the cell. In order to remove all the residual extracellular water component, the pellets were repeatedly washed with deuterated PBS by resuspension and centrifugation. Finally, the cell pellet was spread onto aluminium foil for QENS measurements.

Incoherent Neutron Scattering from Living Cells. In our pellet sample, intracellular $\mathrm{H}_{2} \mathrm{O}$, which has been found to represent ca. $70-80 \%$ of the total cell weight ${ }^{27}$ (i.e., $80 \%$ for neoplastic cells), ${ }^{25}$ was still present. The remaining $20-30 \%$ is therefore related to hydrogenated cell components, with macromolecules usually 
accounting for $>90 \%$ of the dry cell weight. Proteins represent half of this amount (45-50\%) and lipids below 10\% (2-9\%). In addition, the hydrogen content of protein is sensibly higher $(50 \%)$ than that in lipids (30\%). ${ }^{16,28}$ Therefore, essentially, our incoherent signal mostly arises from the intracellular water hydrogen, while most of cellular signal comes from macromolecular dynamics (especially proteins). ${ }^{16,17}$ In regards of $\mathrm{D}_{2} \mathrm{O}$, it only would account for a small residual amount of the extracellular medium, and the incoherent contribution of $\mathrm{D}_{2} \mathrm{O}$ on IRIS is estimated to be smaller than $4 \%$ at $\mathrm{Q}$ $<1.3 \AA^{-1} \cdot{ }^{29}$ Given these considerations, we thus expect that the $\mathrm{D}_{2} \mathrm{O}$ contribution to the total incoherent scattering would be negligible.

QENS Measurements. QENS data were acquired on the backscattering spectrometer IRIS ${ }^{30}$ at the ISIS Pulsed Neutron and Muon Source of the Rutherford Appleton Laboratory (United Kingdom). The measurements were performed using the PG002 analyser that affords a resolution of $17.5 \mu \mathrm{eV}$, a dynamical range from -0.4 to $0.4 \mathrm{meV}$, and a $Q$-range from 0.42 to $1.85 \AA^{-1}$. Indium-sealed annular aluminum cans were used to hold the POPC liposome dispersion, while for measurements on cell pellets, we employed 0.1 mm-thick $(3 \times 5 \mathrm{~cm})$ flat cans (the beam size at the sample being 2.2 $\times 4.4 \mathrm{~cm}$ ) that were oriented at $-30^{\circ}$ with respect to the incident beam. A vanadium sample, which is a purely incoherent elastic scatterer, was measured to define the instrument resolution and correct for detector efficiency. In addition, data were grouped and converted to $S(Q, \omega)$ using the Mantid software. ${ }^{31}$ The data were corrected for buffer scattering by subtracting either the $\mathrm{D}_{2} \mathrm{O}$ or $\mathrm{D}_{2} \mathrm{O}$ / DMSO spectra where necessary, to minimize the scattering from the buffer and highlight any effect of ZIAPIN2 and DMSO on the lipid dynamics only. The transmission of the samples was $\approx 90 \%$, and multiple scattering was neglected. QENS data on liposomes were taken at 283,300, and $310 \mathrm{~K}$ thus above the POPC phase transition $(\sim 271 \mathrm{~K})$, while on HEK cells, the experiment was carried out at 300 $\mathrm{K}$. All the data can be found in reference. ${ }^{32}$

QENS Fundamentals and Data Analysis. In a neutron spectroscopy experiment, we measure the double-differential scattering cross section $\delta^{2} \sigma / \delta E \times \delta \Omega$, which quantifies the probability that a neutron is scattered with an energy change $\delta E$ into the solid angle $\delta \Omega$. When the energy exchange is zero or it falls within the energy resolution of the instrument, the scattering event is called elastic, whereas if $\delta E$ is not zero, the event is called inelastic. In particular, if $\delta E$ is very small as it happens in the case of a wide range of relatively slow motions in soft matter, we observe a broadening of the scattering function around the elastic line and the scattering is known as quasielastic. Such quasi-elastic broadening encodes a variety of dynamical processes including rotations and translations (see below). The measured signal can be mathematically expressed as a function that depends on the exchanged momentum transfer vector $Q$ and energy, the so-called scattering law:

$$
S(\boldsymbol{Q}, \omega)_{\text {measured }}=R(\boldsymbol{Q}, \omega) \otimes S(\boldsymbol{Q}, \omega)
$$

where $R(Q \omega)$ represents the measured instrumental resolution function that is convoluted with the real scattering law. Although the scattering signal is the sum of both coherent and incoherent contributions (defined by the neutron-nucleus interaction), for our system, the signal is dominated by the incoherent scattering of the hydrogenated phospholipids in membranes and cell macromolecules. Therefore, we essentially investigate the dynamics of these systems in the energy range set using our spectrometer. In general, the scattering function can be expressed in terms of three independent contributions, namely, vibrations, rotations, and translations:

$$
S(\boldsymbol{Q}, \omega)=S(\boldsymbol{Q}, \omega)_{\text {vib. }} \otimes S(\boldsymbol{Q}, \omega)_{\text {rot. }} \otimes S(\boldsymbol{Q}, \omega)_{\text {trans. }}
$$

The scattering function can be further expressed as

$$
S(Q, \omega)=A 0(\boldsymbol{Q}) \delta(\omega)+\sum_{i=1}^{2} \frac{A_{i}}{\pi} \frac{\Gamma_{\mathrm{i}}}{\Gamma_{\mathrm{i}}^{2}+\omega^{2}}
$$

where the first term is the the elastic part that contains information on motions slower than the instrumental resolution, and the second term is the so-called quasi-elastic contributions to the total scattering law that can be modeled in terms of Lorentzian functions of different widths, denoted by the half width at half-maximum of the Lorentzian peak (HWHM) $\Gamma$. The Q-dependence of the HWHM (usually expressed as $\Gamma$ vs $Q^{2}$ ) contains detailed information about the dynamics of the system. On the other hand, the elastic contribution gives information about the geometry of the motion via the $A_{0}(Q)$ term, also known as the elastic incoherent structure factor (EISF), which actually represents the space Fourier transform of the average particle distribution taken at infinite time. In our case, the scattering function could be described by the convolution of two Lorentzian curves, each of them associated with different relaxation processes plus an EISF, as expressed below:

$$
\begin{aligned}
S(Q, \omega)= & (A(\boldsymbol{Q}) \delta(\omega)+(1-A(\boldsymbol{Q}))) \frac{\Gamma_{\mathrm{a}}}{\Gamma_{\mathrm{a}}^{2}+\omega^{2}} \otimes B(\boldsymbol{Q}) \delta(\omega) \\
& +(1-B(\boldsymbol{Q}))) \frac{\Gamma_{\mathrm{b}}}{\Gamma_{\mathrm{b}}^{2}+\omega^{2}}
\end{aligned}
$$

Data analysis and fitting were carried out by using the DAVE software $^{33}$ and Mantid routine. ${ }^{31}$

\section{RESULTS AND DISCUSSION}

Effects on Model Membranes. QENS measurements were carried out on POPC liposomes in solution and intact HEK cells to elucidate possible perturbation on phospholipids dynamics brought about by the intramembrane actuator ZIAPIN2 and its vehicle solvent DMSO. The dynamical range of the IRIS spectrometer, in particular, allows investigation of internal motions with characteristic times of few $p s$, as well as slower lateral diffusive motions that occur in tens of $p s .^{34-36}$ Although articifical vesicles made of POPC or simple mixtures of charged/zwitterionic phospholipids do not totally reconstruct the real biological settings, as the cellular membrane usually comprises not only these elements but also proteins and cholesterol-enriched regions, such simple membrane models are widely employed in biophysics to investigate the membrane status as a function of external stimuli (i.e., temperature and drugs, among others) without the interference of other physiological effects occuring in living cells. $^{23,34,35,37-39}$

Starting from the measurements on liposomes in solution, we best-fitted the QENS curves by using one delta function that relates to the elastic scattering and two Lorentzians that account for the quasi-elastic dynamics. In previous investigations on POPC bilayers performed in a comparable timerange, Wanderlingh et al. have employed a three-Lorentzian model, with a broad Lorentzian $(\mathrm{HWHM} \approx 2 \mathrm{meV}$ ) attributed to the motion of hydrogen with respect to the bounding carbon atom ( $0.33 \mathrm{ps})$ and two narrow components related to localized motions, such as lipid chain conformational dynamics $(\approx 0.8 \mathrm{meV})$ and motion of the whole phospholipid molecules $(0.05 \mathrm{meV}){ }^{34,35}$ In analogy with these studies, we assign our first broad component $(0.2-0.6 \mathrm{meV}$, fast in the figure legend) to the phospholipid internal motions, while our narrow Lorentzian can be related to slower diffusive lateral motions (0.04-0.05 meV, slow in the figure legend). ${ }^{36}$ We did not need a third Lorentzian, which would in any case be outside our measurement range. The possible contribution of the liposomes center of mass diffusion to the overall dynamics can be estimated by calculating the self-diffusion coefficient $(D s)$ of the vesicles via the Stokes-Einstein equation, approximating the vesicles as rigid spheres: $D s=k_{\mathrm{B}} T / 6 \pi \eta R_{\mathrm{L}}$, where $k_{\mathrm{B}}$ is the Boltzmann constant, $T$ is the temperature, and $\eta$ is the solvent viscosity (for water, $\eta=8.94 \times 10^{-4} \mathrm{~Pa} \mathrm{~s}$ ). For $100 \mathrm{~nm}$ vesicles, the diffusion coefficient is $4.8 \times 10^{-12} \mathrm{~m}^{2} / \mathrm{s}$. Thus, the root 


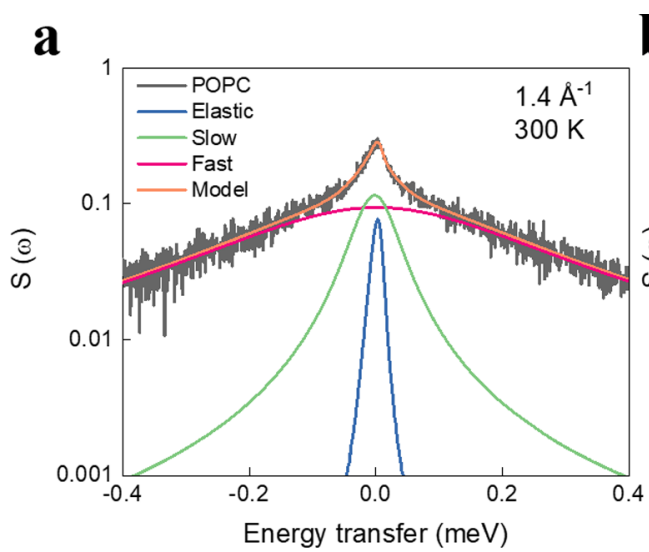

b

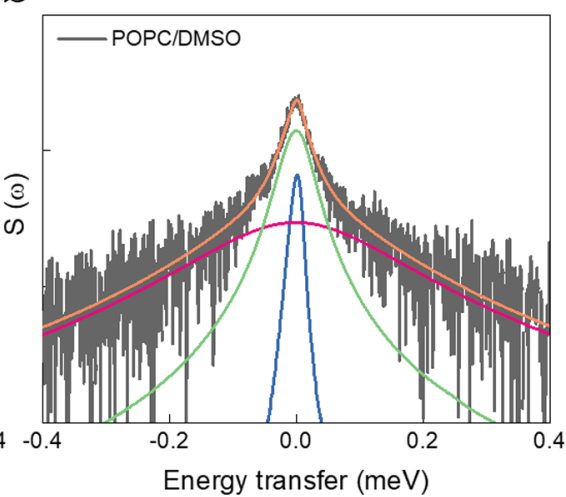

c

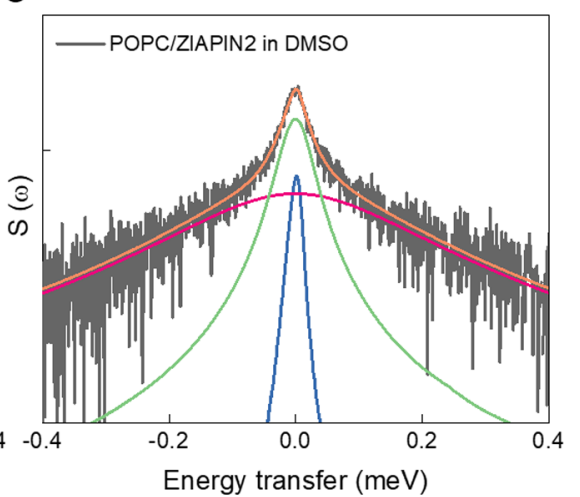

d
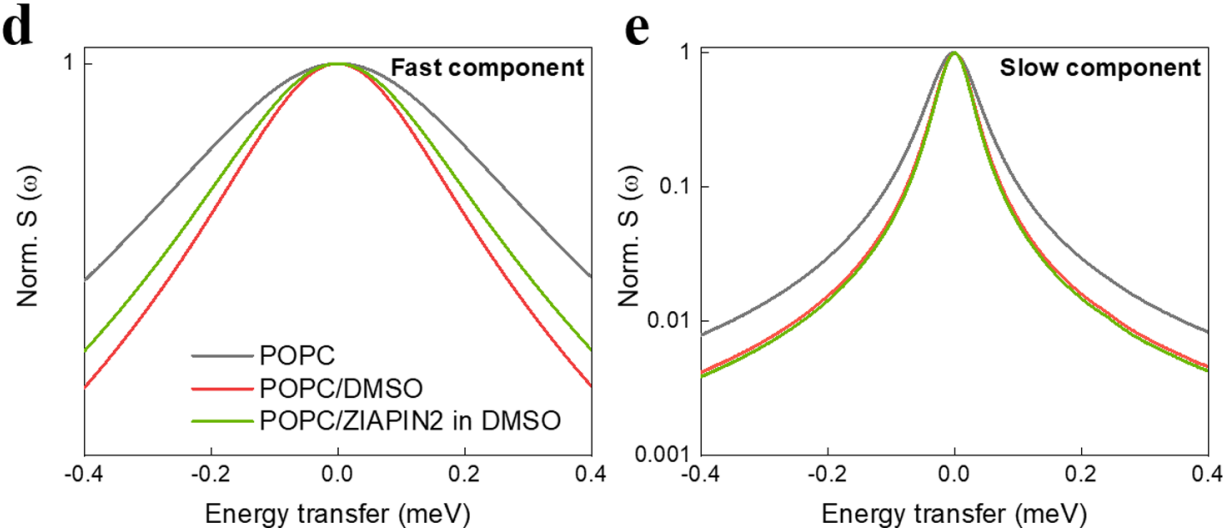

Figure 2. Fitting of the QENS curve for (a) POPC, (b) POPC/DMSO, and (c) POPC/ZIAPIN2. To describe the phospholipids dynamics, we used a delta function that is linked to the elastic scattering that comprises all those motions slower than our resolution. The two Lorentzians account for the quasi-elastic dynamics. In particular, the (d) fast Lorentzian can be attributed to the convolution of internal phospholipid motions, while the (e) slow component to lateral diffusive motions.

mean square $(\mathrm{rms})$ distance $=\sqrt{2 \mathrm{Dt}}$ for $100 \mathrm{~nm}$ vesicles at the probed picosecond time scale is around $3 \mathrm{pm}$, which is two orders of magnitude smaller than our smallest probed length scale $(3.5 \AA)$. Based on this, the contribution from the center of mass diffusion can be neglected in the analysis.

Examples of the fit quality at $Q=1.4 \AA^{-1}$ taken at $300 \mathrm{~K}$ for POPC, POPC/DMSO, and POPC/ZIAPIN2 in DMSO are reported in Figure 2a-c (see Figure S1 for example of fitting at $1.4 \AA^{-1}$ ). We observe already some interesting features in the QENS spectra, namely, (i) a general lowering and narrowing of the QENS signal upon addition of DMSO (Figure 2b); (ii) a partial recovery of the POPC spectral line shape when ZIAPIN2 is internalized in the lipid membrane. Breakdown of the fitting components at $Q=1.4 \AA^{-1}(300 \mathrm{~K})$ for the three samples (Figure $2 \mathrm{~d}, \mathrm{e}$ ) shows that both fast and slow dynamics narrow down in response to the addition of DMSO. On the other hand, interestingly, ZIAPIN2 counteracts the effect of DMSO and re-stabilizes POPC fast dynamics, at least at this length scale and temperature. Note that we do not expect any appreciable change in the membrane fluid phase (POPC $T_{\mathrm{m}}=$ $\left.-2{ }^{\circ} \mathrm{C}\right)^{40}$ within the investigated thermal range and under the three different experimental conditions.

One of the great advantages of the quasi-elastic neutron scattering method lies in the possibility of obtaining dynamical information concurrently with spatial information. To extract detailed parameters, we investigated the dependence of the HWHM $(\Gamma)$ of the Lorentzian components as a function of the momentum transfer $Q$ (Figure 3). The HWHMs are directly related to the characteristic times associated with the motions of the scattering nuclei involved $(\tau=\hbar / \Gamma)$. The fast component reflects dynamics occurring in few picoseconds, which can be attributed to lipid localized internal motions (Figure $3 \mathrm{a}-\mathrm{c}$ ), while the slow component can be connected to lateral diffusive motions (Figure $3 \mathrm{~d}-\mathrm{f}$ ). ${ }^{34,35}$ The $\Gamma_{\text {fast }}$ line shape of pure POPC (Figure 3a) shows a monotonic increase with $Q^{2}$ and flattens toward a finite nonzero value $(0.24 \mathrm{meV}, 2.8$ ps) in the $Q=0$ limit, in agreement with what has been found in previous studies. ${ }^{34,35}$ The existence of a nonzero value is a typical signature of internal localized motions of phospholipids in fluid bilayers ${ }^{14,18,19,36}$ in which the hydrogen atoms diffuse within local spheres as described by the Carpentier model. ${ }^{41}$ In the investigated time window, the dynamics could involve various kinds of motion, including chain reorientation, conformational changes, and methyl group rotations, among others. $^{36}$

A similar picture is obtained upon addition of ZIAPIN2 to the POPC liposomes (Figure 3c), such that the nature of the motions is similar; however, the extrapolated HWHM at $Q=0$ is now lower in energy $(0.13 \mathrm{meV})$ corresponding to a longer relaxation time of 5.2 ps. In general, we note a little dependence on the temperature for POPC and POPC/ ZIAPIN2 samples, possibly owing to the fluid state of the bilayer whose dynamical properties are almost insensitive to temperature variations while being in the same phase. ${ }^{36,42}$ Passing to $\Gamma_{\text {slow }}$ vs momentum transfer, for POPC (Figure 3d), we observe a $Q^{2}$-independent linewidth, ranging from 30-40 $\mu \mathrm{eV}$ and corresponding to correlation times of $16-22$ ps. This can be attributed to slower stochastic reorientations of the lipid 

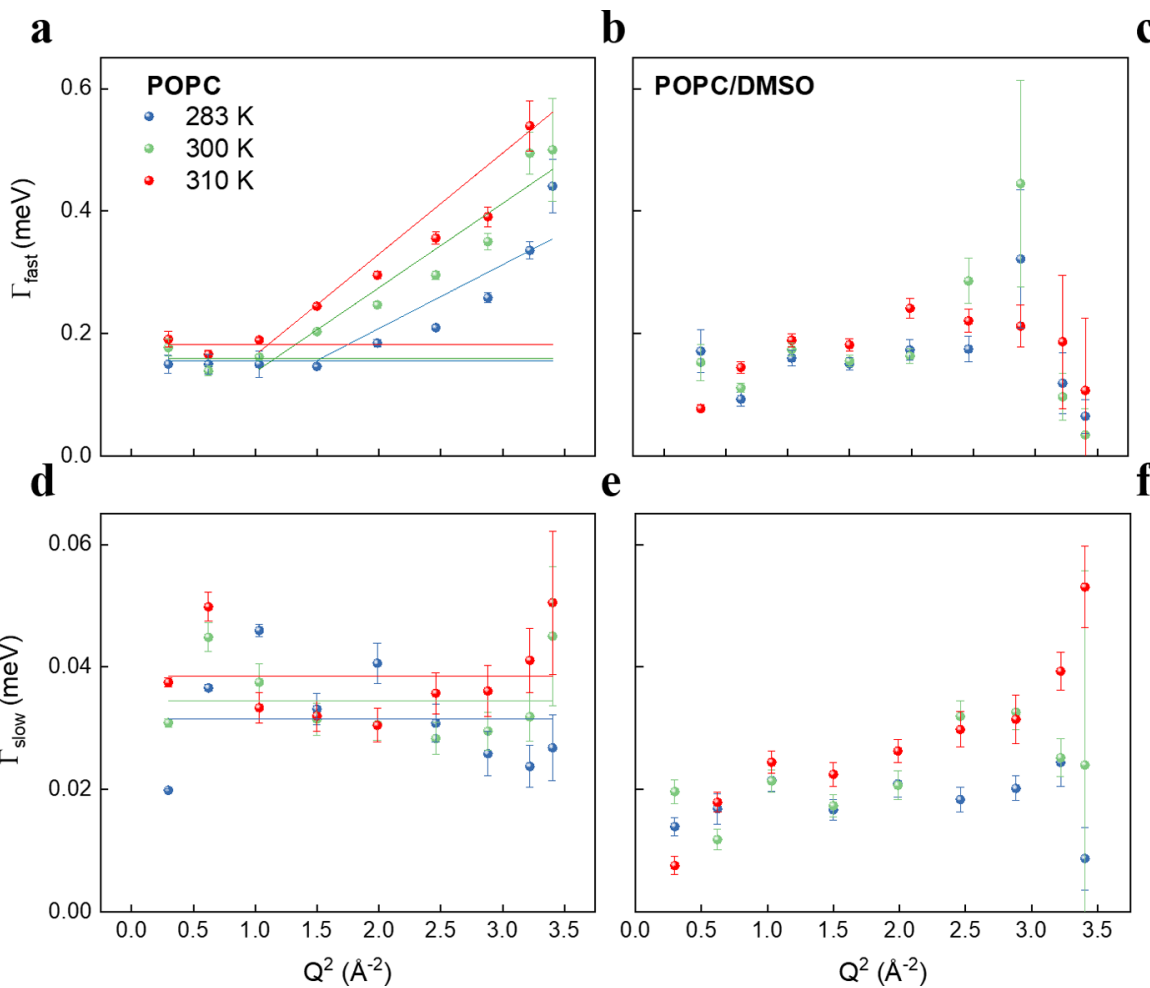

C
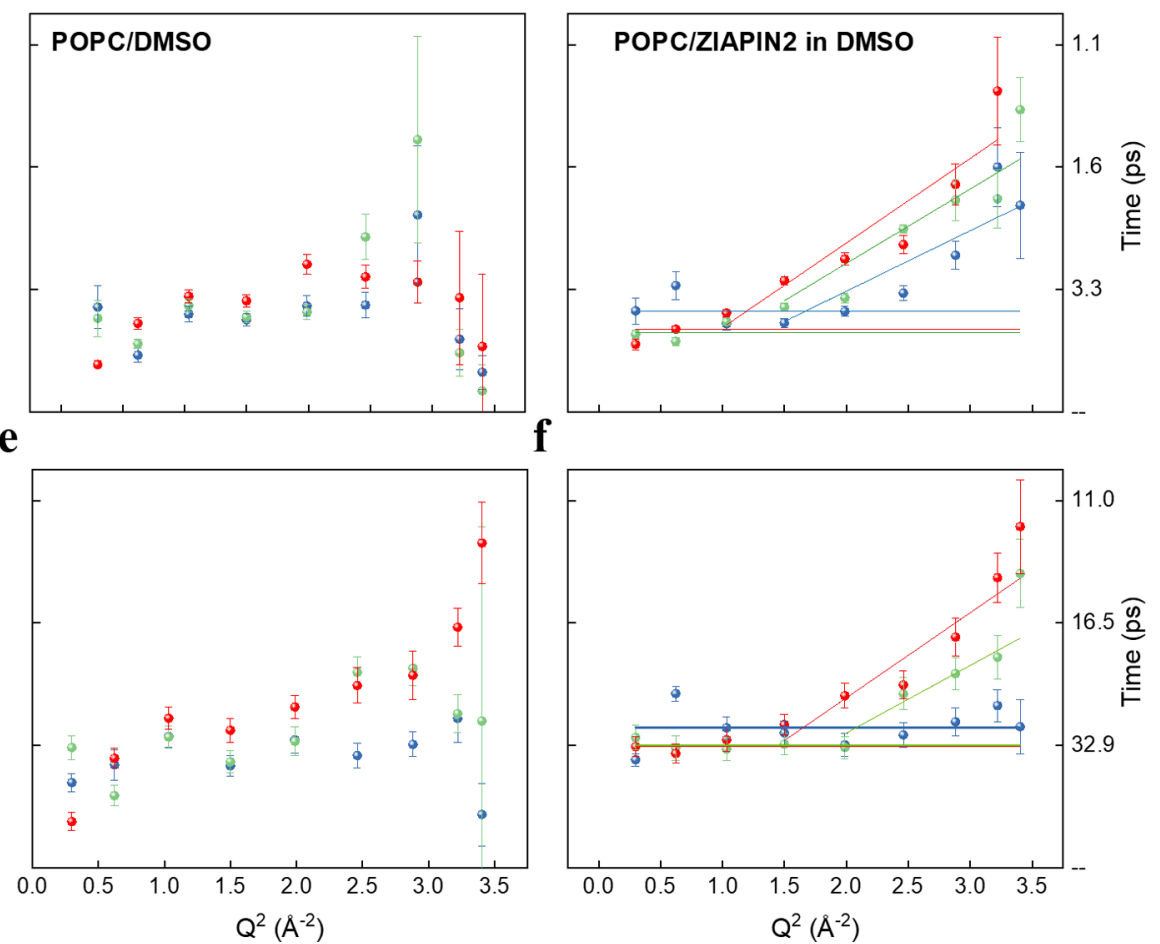

Figure 3. HWHM of the broad Lorentzian $\Gamma_{\text {fast }}$ as a function of $Q^{2}$ for (a) POPC, (b)POPC/DMSO, and (c) POPC/ZIAPIN2. HWHM of the narrow Lorentzian $\Gamma_{\text {slow }}$ as a function of $Q^{2}$ for (d) POPC, (e) POPC/DMSO, and (f) POPC/ZIAPIN2. The solid lines highlight the trend of the HWHM value linewidths. We show more details on the complex trend of the POPC/DMSO sample in the Supplementary Information (Figure S2).

molecular sub-units, for example, fatty chains. ${ }^{16}$ Interestingly, for POPC/ZIAPIN2 (Figure 3f), the $\Gamma_{\text {slow }}$ exhibits a constant linewidth at $283 \mathrm{~K}$, while at higher temperatures, the linewidths suggest a scenario of confined diffusive motions (like that observed in with $\Gamma_{\text {fast }}$ ) with a relaxation time of 30 ps at the $Q=0$ limit.

Instead, the behavior of POPC/DMSO is very different and looks difficult to interpret with standard models. Starting from $\Gamma_{\text {fast }}$ (Figure $3 \mathrm{~b}$ ), the data at all temperatures indicate that internal conformational transitions are strongly reduced with what looks like a long plateau, spanning up to rather large $Q$ values $\left(1.6-1.7 \AA^{-1}\right)$. An alternative picture could be the activation of a jump-diffusion mechanism, which describes the diffusion between sites with residence time at each site $\tau_{0}$ and diffusivity $D$ (see Figure S2). Unfortunately, the data do not render any certainty to the actual mechanism taking place. At higher Q-values, there is an anomalous decrease of the HWHM, suggesting that the effect of the DMSO interaction with the lipids is to highly limit its conformational reorientations at the local scale. This will be discussed in the next paragraphs. For $\Gamma_{\text {slow }}$ (Figure 3e), we see a flat linewidth at 283 and $300 \mathrm{~K}$ with correlation times of $29-37 \mathrm{ps}$. However, at $310 \mathrm{~K}$, the behavior changes. In this case, the data could be rationalized in terms of a jump-diffusional mechanism likely kicking in at the low- $Q$ regime and a linear increase of HWHM at higher- $Q$ values that can be associated with a localized diffusion (see Figure S2 for more details about POPC/DMSO curve fitting). Alternatively, this can also be described as switching to a free lateral diffusion triggered by the rise in temperature while still having very restrained internal reorientations at the local scale (given the results for the fast Lorentzian). We will rationalize the former in terms of DMSO- induced enhancement of the lipid packing that in turns restricts and slows down the fast conformational dynamics (see the next paragraph about EISF analysis), while the latter trend suggests that at the short lengthscales, the slow whole-chain dynamics are still disordered-driven due to the fluid state. The much more complex behavior observed with change at $310 \mathrm{~K}$ for POPC/DMSO could be connected to the stabilization of a mixed sub-gel phase that has been observed via calorimetry and XRD studies for DMSO-treated phosphatidylcholine bilayers. $^{43-45}$ In any case, a more detailed and systematic investigation over the effect of DMSO on the lipid bilayer in model membranes and intact cells can be matter of further studies.

Specifically, the effect of DMSO on model membranes has been studied extensively in the last two decades, owing to its large use as a cryoprotectant in cellular systems and a vehicle solvent for a number of drugs. ${ }^{44,46-51}$ It has been observed that if employed at concentrations $<10 \mathrm{~mol} \%$, which is in line with our concentration of $0.3 \mathrm{~mol} \%$, DMSO tends to dehydrate the membrane by disrupting the dynamic hydrogen-bond water near the lipid membrane surface. ${ }^{50}$ This causes a drastic decrease in the range of the steric hydration repulsion, leading to a reduction of the solvated volume of the phospholipid head groups. $^{44,47}$ Our QENS data seem to capture a rigidification of the bilayer both at the time/length space of internal motions, as we can observe a general increase of the characteristic times and, interestingly, more complex dynamical scenario than the other two samples in which both fluid and rigid dynamical regimes likely coexist (i.e., a sub-gel state). Based on those studies, we therefore attribute such DMSO-induced effects to water dehydration at the bilayer interface. Interestingly, the effect of ZIAPIN2 incorporation in the phospholipid bilayer 
a

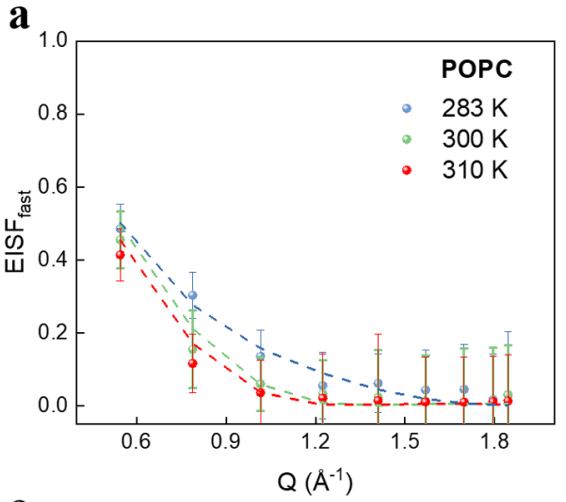

c

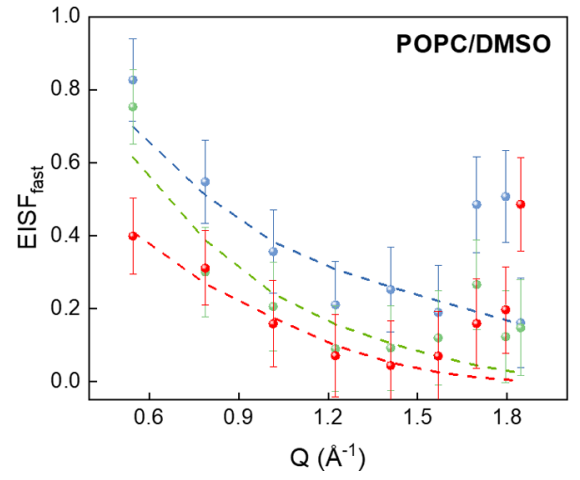

b

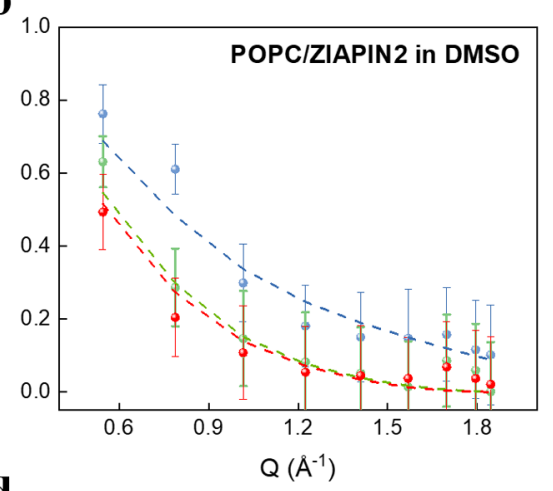

d

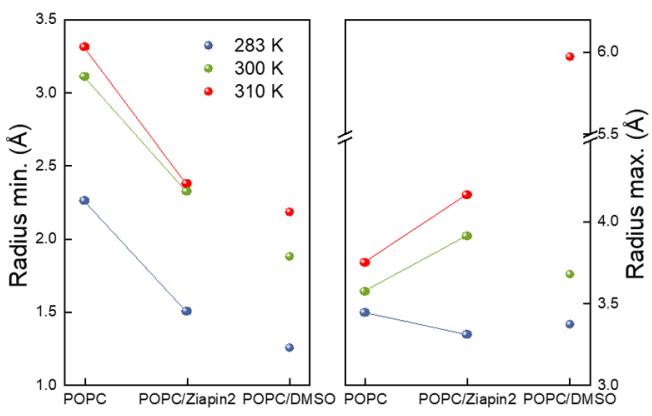

Figure 4. EISF $_{\text {fast }}$ vs $Q$ for (a) POPC, (b) POPC/ZIAPIN2, and (c) POPC/DMSO. The dashed lines represent the fitting model. (d) Minimum and maximum spherical radii of the localized internal motion estimated via the fitting model. In this case, broken lines are used to highlight the different behavior of POPC/DMSO in comparison with the other two samples. Data are presented as a function of temperature.

leads to different effects depending on the time/length scales probed. The recovery of the fast and localized of internal motion can be attributed to the insertion of the positively charged pyridine rings at the level of the lipid heads. ${ }^{23}$ This, in turns, restores the solvation volume of the phosholipids head group that was previously reduced due to DMSO dehydration. The slow dynamics, instead, diverge from stochastic molecular reorientations found mainly in pristine POPC, showing evidence of localized diffusional motions. We interpret this in terms of higher dynamical disorder in POPC/ZIAPIN2 at the lengthscale of molecular diffusions, due to the incorporation of the ZIAPIN2 apolar backbone in the central region of the bilayer and dimerization with the molecules anchored in the opposite part of the leaftlet, as found in our previous study. ${ }^{23}$ Taken together, these effects concur to mitigate the membrane rigidification brought about by DMSO, as evidenced by the broader QENS signal of the POPC/ZIAPIN2 systems compared to POPC/DMSO (see Figure $2 b, c)$. To further validate these data, we performed experiments on POPC/ZIAPIN2 at a lower concentration ( $5 \mu \mathrm{M}$, Figure S3). Here, we note a narrower QENS signal than at $25 \mu \mathrm{M}$, thus clearly denoting a less effective action of ZIAPIN2 in softening the lipid membrane.

To obtain insights into the geometry of motion, we analysed the dependency of the elastic incoherent structure factor (EISF) on the momentum transfer. In particular, the internal motion that is localized in character can be modeled as a linear combination of the elastic component $A_{0}(Q)$ and the Lorentzian function as reported in eq 3. We made use of an analytical expression for the EISF, which describes the dynamics along the phospholipid molecules as localized diffusions within spheres of different radii. This method has been successfully employed to describe the motion in a variety of systems, such as lyophilized lipids, ${ }^{52}$ micelles, ${ }^{53}$ and phospholipid bilayers. ${ }^{19,36,54}$ The model can be represented by the following expression:

$$
A 0(\boldsymbol{Q})=\frac{1}{N} \sum_{n=1}^{N}\left[\frac{3 j_{\mathrm{i}}\left(Q R_{\mathrm{n}}\right)}{Q R_{\mathrm{n}}}\right]^{2}
$$

where $j_{\mathrm{i}}$ is the first-order spherical Bessel function and $R$ is the spherical radius. In our case, we best-fitted the EISF by using $N$ $=2$ to take into account the different diffusivities at the lipid head group and lipid chain levels, ${ }^{54}$ while a further increase of the number of radii did not improve the fit quality. The EISF of the fast internal motion vs $Q$ is displayed in Figure $4 a-c$ for POPC, POPC/DMSO, and POPC/ZIAPIN2, respectively. We can note three important effects on the EISF: (i) a higher elastic fraction intensity for POPC/ZIAPIN2 than pristine POPC, alongside a relatively faster decay of the EISF line in this latter sample; (ii) DMSO clearly increases the elastic fraction in comparison to pristine POPC and POPC/ ZIAPIN2; (iii) an enhancement of the elastic fraction at the higher $Q$-values (at $\approx 1.8 \AA^{-1}$ ) for DMSO that in fact cannot be captured by the analytical model. Since this length scale $(d-$ space $=3.1-3.8 \AA)$ is compatible with the lateral chain packing in bilayers, ${ }^{55}$ we essentially observe the influence of DMSO on the lipid tail arrangement. In particular, it has been demonstrated that DMSO leads to a dehydration of the intermembrane space, thus stabilizing the lipid phase. ${ }^{46,47}$ Therefore, the EISF for POPC/DMSO encodes both a general dynamics retardation and, specifically, a reduction of proton motility in the lipid phase. Note that this would explain the anomalous decrease of the HWHM linewidth between 1.5-1.8 
POPC: Disordered motions

- Internal localized motions $(2-3 \AA ; 1-3 \mathrm{ps})$;

- Lateral motions (16-20 ps)
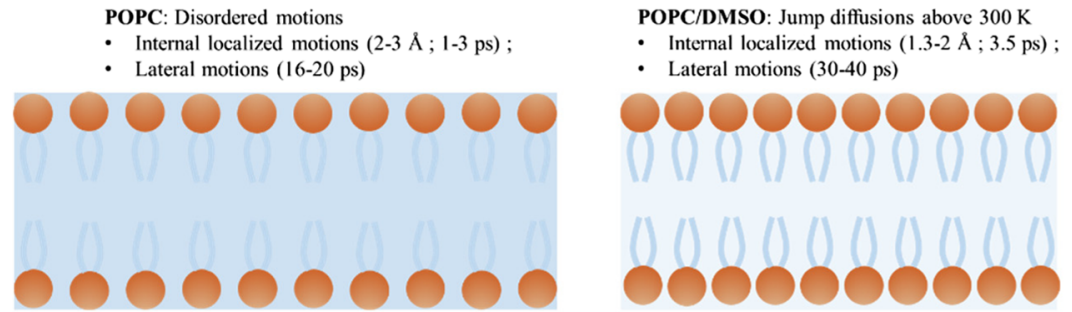

DMSO dehydration $\rightarrow$ Membrane rigidification
POPC/ZIAPIN2: Disordered motions

- Internal localized motions (1.5-2.4 $\AA$; 1-5 ps) ;

- Lateral motions (11-30 ps)

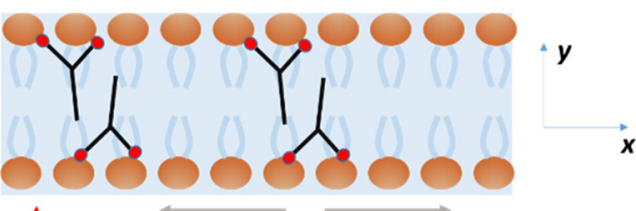

ZIAPIN2 intercalation $\rightarrow$ Membrane relaxation

Figure 5. Pictorial representation of the dynamical scenario caused by DMSO and ZIAPIN2 addition to the POPC liposomes. In the pristine status, POPC dynamics can be described by localized disordered internal motions and slower lateral motions. Addition of DMSO leads to a rigidification of the bilayer dynamics and a more ordered dynamical scenario, as highlighted by the kicking in of jump-diffusional motion at $310 \mathrm{~K}$. Based on a previous literature study, this can be attributed to dehydration in the close proximity of the bilayer, which would substantially decrease the solvation volume and restrict the geometry of motion. Eventually, addition of ZIAPIN tends to re-establish the POPC dynamics, likely due to re-solvation of the lipid heads and to the expansion of the bilayer along the $x$ direction. We attribute this latter effect to the reduction of the bilayer thickness that we have previously linked to ZIAPIN2 trans-dimerization in the dark and to the consequent expansion in the other direction owing to the incompressibility of the membrane.
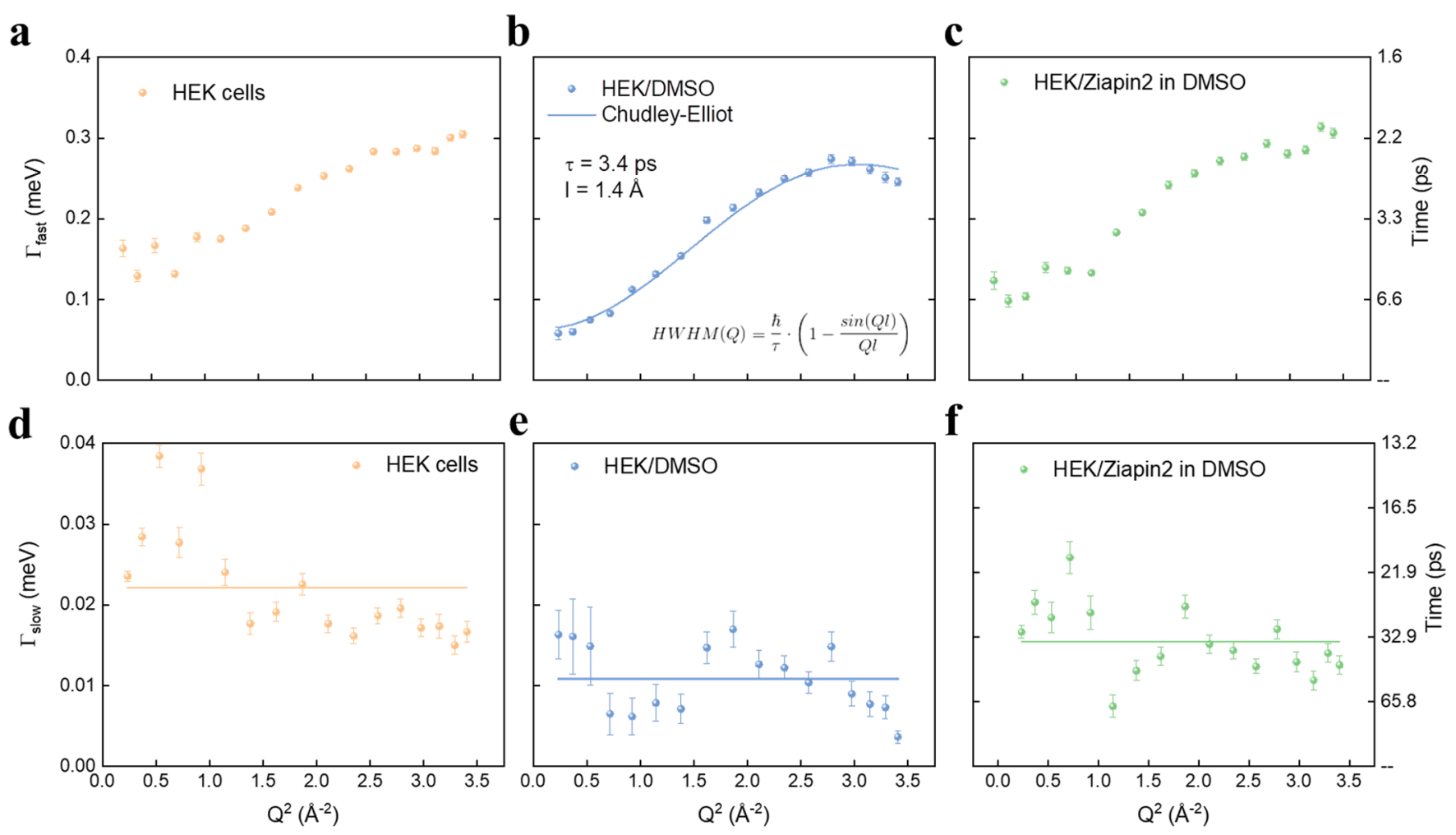

Figure 6. HWHM of the broad Lorentzian $\Gamma_{\text {fast }}$ as a function of $Q^{2}$ for (a) HEK at $300 \mathrm{~K}$, (b) HEK/DMSO, and (c) HEK/ZIAPIN2. HWHM of the narrow Lorentzian $\Gamma_{\text {slow }}$ as a function of $Q^{2}$ for (d) HEK, (e) HEK/DMSO, and (f) HEK/ZIAPIN2. The solid lines represent either the fitting or the average HWHM values.

$\AA^{-1}$ in POPC/DMSO (see Figure 3b,e), which can be indeed related to the rigidification and consequent increase of the characteristic time brought about by DMSO dehydration. Interestingly, ZIAPIN2 addition causes a reduction of the elastic signal excess in the $1.5-1.8 \AA^{-1}$ region when compared to POPC/DMSO. If we look at the estimated minimum and maximum radii (Figure $4 \mathrm{~d}$ ), we observe that DMSO addition leads to a reduction of $R_{\min }$ and $R_{\max }$, with the exception for the marked increase $R_{\max }$ at $310 \mathrm{~K}$. We hypothesize that this can be related to either a fitting artifact or to the kicking in of another dynamical scenario at the low- $Q$ region at $310 \mathrm{~K}$, i.e., the abomentioned sub-gel phase.
The EISF vs $Q$ for the narrower Lorentzian (EISF slow $_{\text {, Figure }}$ S4) shows that while in the POPC sample, the EISF $_{\text {slow }}$ contribution to the total scattering is virtually flat, in POPC/ DMSO and POPC/ZIAPIN2, there is a clear Q-dependence and relatively higher signals than the POPC pristine. This can be attributed to the higher diffusive motion localization in these latter samples due to the DMSO-induced dynamical restriction.

Coupling the results from the HWHM trends and the EISF data, we propose the following scenario that the action of ZIAPIN2 on the bilayer is in fact systemic, involving both a strong interaction with the lipid heads and with the acylic part of the bilayer, rendering the lipid membrane more relaxed and 
dynamically flexible. These data corroborate and nicely complement the structural information obtained in our previous investigations, ${ }^{23,24}$ indicating that the effective ZIAPIN2 electrophysiologic action lies on the formation of membrane-spanning dimers that lead to a reduction of the bilayer thickness. We speculate here that while the solvation of the phosholipid heads can be attributed to the electrostatic interaction with the positively charged pyridine rings, the relaxation at the lipid tail level can be instead linked to an expansion of the bilayer along the $x$ direction (Figure 5) as a result of the membrane thickness shrinkage along the $y$ direction, due to the incompressibility of the lipid membrane. ${ }^{56}$

Effects on HEK Cells. Having arrived at a dynamical picture using model membranes, we analyse the QENS data measured real cells, in order to confirm whether a similar picture is observed in highly complex biological specimens. Like for the model membranes, data were best-fitted with two Lorentzians used to describe two distinct dynamical processes (Figure S5). Although in these complex samples the scattering signal will be an average of hydrogens from intracellular water and from a wide variety of structures in the cells including proteins, organelles, and lipids among others (see the Experimental Section) and thus an average of multiple dynamical processes, ${ }^{16,17,57}$ we can see that the linewidths follow virtually the same behavior observed for POPC liposomes, in terms of the addition of DMSO or ZIAPIN2, at least from the qualitative point of view. For instance, the spectra shown in Figure $S 3$ (at $Q=1.4 \AA^{-1}$ ) report a clear narrowing down of the QENS signal upon addition of DMSO. The data show a broadening due to ZIAPIN2, which again suggests that ZIAPIN2 leads to enhancement of membrane flexibilty once partitioned in the bilayer. To obtain information about the nature of the motion in the time/space domains, we investigated the dependence of the HWHM $(\Gamma)$ of the Lorentzian components as a function of the momentum transfer also for HEK cells (Figure 6). Starting from $\Gamma_{\text {fast }}$ (Figure $6 \mathrm{a}-\mathrm{c}$ ), we see that in general the linewidths follow the typical behavior of localized jump-diffusion with data saturating at the high $Q^{2}$-values, while the HWHM tends at a constant value in the low $Q^{2}$-region that indicates confining effects of local boundaries. ${ }^{57}$ For pristine HEK cells, we see that the plateau extends to $Q^{2}=1.5 \AA^{-2}$, while HWHM saturates at around $0.3 \mathrm{meV}(2.2 \mathrm{ps})$. The addition of DMSO leads to a general lowering of the linewidth, suggesting also in this case a retardation of the dynamics. However, we can note two important effects, specifically a less extended plateau at the low- $Q$ region, if compared with pristine cells, and a decrease of the linewidth at the high- $Q$ region. The former case can be explained in terms of less constrained motion, likely owing to the higher dynamical order in DMSO-treated cells and in analogy with what we observed in POPC/DMSO. In this case, interestingly, the linewidth can also suggest the kicking in of a different mechanism, specifically the Chudley-Elliot model that describes diffusional motions occurring via discrete jumps between sites in a lattice (see Figure 6b inset), a typical dynamical scenario of those systems exhibiting a short range order. $^{58}$ Conversely, ZIAPIN2 leads to a total restoration $\Gamma_{\text {fast }}$ trend of pristine cells, a result that is in line with POPC model membranes and strongly indicates a disruption of the DMSOinduced ordering. Passing to $\Gamma_{\text {slow, }}$ we note essentially a $Q^{2}$ independent profile typical of reorientational motions in all three samples. The average HWMH values reflect the findings in liposome, namely, a slowing down of the lateral dynamics upon DMSO addition and a partial recovery of the pristine linewidth after ZIAPIN2 internalization in the lipid membrane (average $\mathrm{HWHM}=2.2 \mu \mathrm{eV}$ (30 ps), $1.1 \mu \mathrm{eV}$ (60 ps) and 1.9 $\mu \mathrm{eV}$ (35 ps), for HEK, HEK/DMSO, and HEK/ZIAPIN2, respectively). The EISF vs $Q$ for the internal motion is reported in Figure S6. Here, we see a reduction of the EISF (increase of $R_{\min }$ ) at the low- $Q$ values for HEK/DMSO, a result that can be ascribed also in this case to the activation of a more spatially extended jump-diffusional mechanism for this sample.

In regards of ZIAPIN2 and DMSO localization in HEK293 cells, in our previous studies, ${ }^{23,24}$ we found that more than $70 \%$ of Ziapin2 was localized to the neuronal surface, with a coverage of $\sim 15 \%$ of the total membrane surface that progressively decreased over time $\left(t_{1 / 2}=36.4 \mathrm{~h}\right.$, confocal microscopy data). The occurrence of fluorescence resonance energy transfer between ZIAPIN2 and the membrane dye CellMask further demonstrates that the molecule is strictly localized to the membrane bilayer. As ZIAPIN2 was internalized right before the experiment, we expect that only a small fraction (around 4-5\%, see supplementary Figure 7a of reference ${ }^{23}$ ) would possibly leave the cell membrane and distribute between the intracellular and intercellular space during the experiment $(8 \mathrm{~h})$. The amphiphilic nature of DMSO would also lead to its localization in the close proximity of the plasma membrane, as it has been already demonstrated in a number of investigations. However, at the moment, we could not rule out completely any permeation inside the cytoplasmic space and interaction with the intracellular water.

Interestingly, however, these data indicate that despite the many dynamical processes contributing to the QENS signal in HEK cells and the relatively small contribution of the lipid membrane component to the total scattering, we still could observe a similar dynamical trend upon ZIAPIN2 and DMSO addition between POPC liposomes and living cells. Further dedicated investigations on contrast matched samples are necessary to address specifically and carefully the phenomena occurring in the plasma membrane of intact cells.

\section{- CONCLUSIONS}

In summary, we have reported a detailed QENS investigation over the dynamics of lipid membranes modified using a new membrane actuator, namely, ZIAPIN2, and its vehicle solvent DMSO. We carried out experiments on both model membranes composed of POPC liposomes in solution and intact HEK cells. Our data suggest strongly that ZIAPIN2 causes a general softening of the bilayer, most probably due to ZIAPIN2/POPC interaction at the lipid head and acylic tail levels. The addition of DMSO leads to a much more complex dynamical picture and one of a more dynamically ordered landscape, likely due to interface dehydration and overall membrane rigidification. The associated change in the solvation volume of the lipid heads and the consequent geometry modification of the plasma membrane (Figure 5) offer an explanation for the observed change in the electrical capacitance of the plasma membrane observed in our previous investigations. $^{23,24}$ Upon addition of ZIAPIN2, the cell electrical capacitance is increased. Based on the simple plane capacitor model $\left(C=\varepsilon \frac{A}{d}\right)$, this is perfectly consistent with the increased surface and reduced thickness we observed. Finally, the slight reduction in capacitance observed upon adding DMSO only is here rationalized; in this case, our data predict a 
smaller surface, due to dehydration. In general, the effects of guest molecules on the overall plasticity of the plasma membrane can be of paramount interest for the design of novel membrane-targeted drugs, e.g., for antibiotics and therapeutics.

\section{ASSOCIATED CONTENT}

\section{SI Supporting Information}

The Supporting Information is available free of charge at https://pubs.acs.org/doi/10.1021/acs.langmuir.0c01846.

Fitting of the QENS curve for POPC liposomes and HEK samples at $1 \AA^{-1}$, fitting of $\Gamma_{\text {fast }}$ and $\Gamma_{\text {slow }}$ with a jump-diffusional model for POPC/DMSO at $310 \mathrm{~K}$, QENS spectrum taken at $300 \mathrm{~K}$ and $Q=0.5 \AA^{-1}$ for POPC liposomes at $5 \mu \mathrm{M}, \mathrm{EISF}_{\text {slow }}$ vs $Q$ for POPC, POPC/DMSO, and POPC/ZIAPIN2, and fitting of the QENS curve for HEK cells at $1.4 \AA^{-1}$, EISF $_{\text {fast }}$ vs $Q$ for HEK samples at $300 \mathrm{~K}$ (PDF)

\section{AUTHOR INFORMATION}

\section{Corresponding Authors}

Giuseppe M. Paternò - Center for Nano Science and Technology, Istituto Italiano di Tecnologia, 20133 Milano, Italy; (1) orcid.org/0000-0003-2349-566X;

Email: giuseppe.paterno@iit.it

Guglielmo Lanzani - Center for Nano Science and Technology, Istituto Italiano di Tecnologia, 20133 Milano, Italy; Dipartimento di Fisica, Politecnico di Milano, 20133 Milano, Italy; (1) orcid.org/0000-0002-2442-4495;

Email: guglielmo.lanzani@iit.it

\section{Authors}

Gaia Bondelli - Center for Nano Science and Technology, Istituto Italiano di Tecnologia, 20133 Milano, Italy

Victoria Garcia Sakai - ISIS Pulsed Neutron and Muon Source, Science and Technology Facilities Council, Rutherford Appleton Laboratory, Didcot OX11 OQX, U.K.

Valentina Sesti - Dipartimento di Chimica, Materiali e Ingegneria Chimica "Giulio Natta", Politecnico di Milano, 20133 Milano, Italy

Chiara Bertarelli - Dipartimento di Chimica, Materiali e Ingegneria Chimica "Giulio Natta", Politecnico di Milano, 20133 Milano, Italy

Complete contact information is available at:

https://pubs.acs.org/10.1021/acs.langmuir.0c01846

\section{Notes}

The authors declare no competing financial interest.

\section{ACKNOWLEDGMENTS}

We acknowledge Fondazione Cariplo for the financial support (grant $\mathrm{n}^{\circ} 2018-0979$ and grant $\mathrm{n}^{\circ}$ 2018-0505). We thank the ISIS Facility, STFC Rutherford Appleton Laboratory for access to neutron beam facilities and to the Research Complex at Harwell (cell culture labs). The authors are grateful to Ms. Sarah Langham and Mrs. Asha Lata Dopplapudi for the technical support at the Research Complex Harwell, to Dr. Francesco Lodola for the help during the cell culture steps and to Prof. Maria Paula Marques and Dr. Ana Batista de Carvalho for the helpful scientific discussions.

\section{REFERENCES}

(1) Schweizer, F. Cationic Amphiphilic Peptides with CancerSelective Toxicity. Eur. J. Pharmacol. 2009, 625, 190-194.

(2) Findlay, B.; Zhanel, G. G.; Schweizer, F. Cationic Amphiphiles, a New Generation of Antimicrobials Inspired by the Natural Antimicrobial Peptide Scaffold. Antimicrob. Agents Chemother. 2010, 54, 4049-4058.

(3) Wang, B.; Wang, M.; Mikhailovsky, A.; Wang, S.; Bazan, G. C. A Membrane-Intercalating Conjugated Oligoelectrolyte with HighEfficiency Photodynamic Antimicrobial Activity. Angew. Chem., Int. Ed. 2017, 56, 5031-5034.

(4) Wang, B.; Queenan, B. N.; Wang, S.; Nilsson, K. P. R.; Bazan, G. C. Precisely Defined Conjugated Oligoelectrolytes for Biosensing and Therapeutics. Adv. Mater. 2019, 31, 1-21.

(5) Seddon, A. M.; Casey, D.; Law, R. V.; Gee, A.; Templer, R. H.; Ces, O. Drug Interactions with Lipid Membranes. Chem. Soc. Rev. 2009, 38, 2509-2519.

(6) Almeida, M. M.; Perez, K. R.; Faig, A.; Uhrich, K. E.; Riske, K. A. Location of the Positive Charges in Cationic Amphiphiles Modulates Their Mechanism of Action against Model Membranes. Langmuir 2019, 35, 14117-14123.

(7) Mitra, M.; Asad, M.; Kumar, S.; Yadav, K.; Chaudhary, S.; Bhavesh, N. S.; Khalid, S.; Thukral, L.; Bajaj, A. Distinct Intramolecular Hydrogen Bonding Dictates Antimicrobial Action of Membrane-Targeting Amphiphiles. J. Phys. Chem. Lett. 2019, 10, 754-760.

(8) Squires, G. L. Introduction to the Theory of Thermal Neutron Scattering; Cambridge University Press: Cambridge, 2012; Vol. 9781107644. DOI: $10.1017 / \mathrm{CBO} 9781139107808$.

(9) Sears, V. F. Neutron Scattering Lengths and Cross Sections. Neutron News 1992, 3, 26

(10) Sakai, V. G.; Arbe, A. Quasielastic Neutron Scattering in Soft Matter. Curr. Opin. Colloid Interface Sci. 2009, 14, 381-390.

(11) García Sakai, V.; Alba-Simionesco, C. Dynamics of Soft Matter; García Sakai, V., Alba-Simionesco, C., Chen, S.-H., Eds.; Neutron Scattering Applications and Techniques; Springer US: New York, NY, 2012. DOI: $10.1007 / 978-1-4614-0727-0$.

(12) Gardner, J. S.; Ehlers, G.; Faraone, A.; García Sakai, V. HighResolution Neutron Spectroscopy Using Backscattering and Neutron Spin-Echo Spectrometers in Soft and Hard Condensed Matter. Nat. Rev. Phys. 2020, 103.

(13) König, S.; Pfeiffer, W.; Bayerl, T.; Richter, D.; Sackmann, E. Molecular Dynamics of Lipid Bilayers Studied by Incoherent QuasiElastic Neutron Scattering. J. Phys. II 1992, 2, 1589-1615.

(14) Sharma, V. K.; Mitra, S.; Mukhopadhyay, R. Dynamic Landscape in Self-Assembled Surfactant Aggregates. Langmuir 2019, $35,14151-14172$.

(15) Gerelli, Y.; Sakai, V. G.; Ollivier, J.; Deriu, A. Conformational and Segmental Dynamics in Lipid-Based Vesicles. Soft Matter 2011, 7, 3929.

(16) Jasnin, M.; Moulin, M.; Haertlein, M.; Zaccai, G.; Tehei, M. In Vivo Measurement of Internal and Global Macromolecular Motions in Escherichia Coli. Biophys. J. 2008, 95, 857-864.

(17) Zaccai, G. Molecular Dynamics in Cells: A Neutron View. Biochim. Biophys. Acta - Gen. Subj. 2020, 1864, 129475.

(18) Sharma, V. K.; Mamontov, E.; Tyagi, M.; Qian, S.; Rai, D. K.; Urban, V. S. Dynamical and Phase Behavior of a Phospholipid Membrane Altered by an Antimicrobial Peptide at Low Concentration. J. Phys. Chem. Lett. 2016, 7, 2394-2401.

(19) Sharma, V. K.; Mamontov, E.; Tyagi, M.; Urban, V. S. Effect of $\alpha$-Tocopherol on the Microscopic Dynamics of Dimyristoylphosphatidylcholine Membrane. J. Phys. Chem. B 2016, 120, 154-163.

(20) Sharma, V. K.; Mamontov, E.; Anunciado, D. B.; O’Neill, H.; Urban, V. S. Effect of Antimicrobial Peptide on the Dynamics of Phosphocholine Membrane: Role of Cholesterol and Physical State of Bilayer. Soft Matter 2015, 11, 6755-6767.

(21) Sharma, V. K.; Mamontov, E.; Ohl, M.; Tyagi, M. Incorporation of Aspirin Modulates the Dynamical and Phase 
Behavior of the Phospholipid Membrane. Phys. Chem. Chem. Phys. 2017, 19, 2514-2524.

(22) Lautner, L.; Pluhackova, K.; Barth, N. K. H.; Seydel, T.; Lohstroh, W.; Böckmann, R. A.; Unruh, T. Dynamic Processes in Biological Membrane Mimics Revealed by Quasielastic Neutron Scattering. Chem. Phys. Lipids 2017, 206, 28-42.

(23) DiFrancesco, M. L.; Lodola, F.; Colombo, E.; Maragliano, L.; Bramini, M.; Paternò, G. M.; Baldelli, P.; Serra, M. D.; Lunelli, L.; Marchioretto, M.; Grasselli, G.; Cimò, S.; Colella, L.; Fazzi, D.; Ortica, F.; Vurro, V.; Eleftheriou, C. G.; Shmal, D.; Maya-Vetencourt, J. F.; Bertarelli, C.; Lanzani, G.; Benfenati, F. Neuronal Firing Modulation by a Membrane-Targeted Photoswitch. Nat. Nanotechnol. 2020, 15, 296-306.

(24) Paternò, G. M.; Colombo, E.; Vurro, V.; Lodola, F.; Cimò, S.; Sesti, V.; Molotokaite, E.; Bramini, M.; Ganzer, L.; Fazzi, D.; D’Andrea, C.; Benfenati, F.; Bertarelli, C.; Lanzani, G. Membrane Environment Enables Ultrafast Isomerization of Amphiphilic Azobenzene. Adv. Sci. 2020, 7, 1903241.

(25) Marques, M. P. M.; Batista De Carvalho, A. L. M.; Sakai, V. G.; Hatter, L.; Batista De Carvalho, L. A. E. Intracellular Water-an Overlooked Drug Target? Cisplatin Impact in Cancer Cells Probed by Neutrons. Phys. Chem. Chem. Phys. 2017, 19, 2702-2713.

(26) Batista De Carvalho, A. L. M.; Mamede, A. P.; Dopplapudi, A.; Garcia Sakai, V.; Doherty, J.; Frogley, M.; Cinque, G.; Gardner, P.; Gianolio, D.; Batista De Carvalho, L. A. E.; Marques, M. P. M. Anticancer Drug Impact on DNA-a Study by Neutron Spectroscopy Coupled with Synchrotron-Based FTIR and EXAFS. Phys. Chem. Chem. Phys. 2019, 21, 4162-4175.

(27) Delpire, E.; Gagnon, K. B. Water Homeostasis and Cell Volume Maintenance and Regulation. Curr. Top. Membr. 2018, 81, 3.

(28) Madigan, M. T.; Martinko, J. M.; Parker, J. Brock - Biology of Microorganisms, 9th Edition. Prentice-Hall 2000.

(29) Stadler, A. M.; Digel, I.; Artmann, G. M.; Embs, J. P.; Zaccai, G.; Büldt, G. Hemoglobin Dynamics in Red Blood Cells: Correlation to Body Temperature. Biophys. J. 2008, 95, 5449-5461.

(30) Carlile, C. J.; Adams, M. A. The Design of the IRIS Inelastic Neutron Spectrometer and Improvements to Its Analysers. Phys. B Phys. Condens. Matter 1992, 182, 431-440.

(31) Arnold, O.; Bilheux, J. C.; Borreguero, J. M.; Buts, A.; Campbell, S. I.; Chapon, L.; Doucet, M.; Draper, N.; Ferraz Leal, R.; Gigg, M. A.; Lynch, V. E.; Markvardsen, A.; Mikkelson, D. J.; Mikkelson, R. L.; Miller, R.; Palmen, K.; Parker, P.; Passos, G.; Perring, T. G.; Peterson, P. F.; Ren, S.; Reuter, M. A.; Savici, A. T.; Taylor, J. W.; Taylor, R. J.; Tolchenov, R.; Zhou, W.; Zikovsky, J. Mantid - Data Analysis and Visualization Package for Neutron Scattering and $\mu$ SR Experiments. Nucl. Instruments Methods Phys. Res. Sect. A Accel. Spectrometers, Detect. Assoc. Equip. 2014, 764, 156.

(32) Paternò, G. M.; Bondelli, G.; Lodola, F.; Garcia Sakai, V. Quasi Elastic Neutron Scattering Study on Photoresponsive Cell Membranes. STFC ISIS Neutron Muon Source 2019. DOI: 10.5286/ ISIS.E.RB1910283.

(33) Azuah, R. T.; Kneller, L. R.; Qiu, Y.; Tregenna-Piggott, P. L. W.; Brown, C. M.; Copley, J. R. D.; Dimeo, R. M. DAVE: A Comprehensive Software Suite for the Reduction, Visualization, and Analysis of Low Energy Neutron Spectroscopic Data. J. Res. Natl. Inst. Stand. Technol. 2009, 114, 341-358.

(34) Wanderlingh, U.; D’Angelo, G.; Branca, C.; Conti Nibali, V.; Trimarchi, A.; Rifici, S.; Finocchiaro, D.; Crupi, C.; Ollivier, J.; Middendorf, H. D. Multi-Component Modeling of Quasielastic Neutron Scattering from Phospholipid Membranes. J. Chem. Phys. 2014, 140, 174901.

(35) Wanderlingh, U.; Branca, C.; Crupi, C.; Nibali, V. C.; La Rosa, G.; Rifici, S.; Ollivier, J.; D’Angelo, G. Molecular Dynamics of POPC Phospholipid Bilayers through the Gel to Fluid Phase Transition: An Incoherent Quasi-Elastic Neutron Scattering Study. J. Chem. 2017, 2017, 1-8.

(36) Dubey, P. S.; Srinivasan, H.; Sharma, V. K.; Mitra, S.; Sakai, V. G.; Mukhopadhyay, R. Dynamical Transitions and Diffusion Mechanism in DODAB Bilayer. Sci. Rep. 2018, 8, 1862.
(37) Ainalem, M. L.; Campbell, R. A.; Khalid, S.; Gillams, R. J.; Rennie, A. R.; Nylander, T. On the Ability of PAMAM Dendrimers and Dendrimer/DNA Aggregates to Penetrate POPC Model Biomembranes. J. Phys. Chem. B 2010, 114, 7229-7244.

(38) Bouvrais, H.; Méléard, P.; Pott, T.; Jensen, K. J.; Brask, J.; Ipsen, J. H. Softening of POPC Membranes by Magainin. Biophys. Chem. 2008, 137, 7-12.

(39) Gupta, S.; De Mel, J. U.; Schneider, G. J. Dynamics of Liposomes in the Fluid Phase. Curr. Opin. Colloid Interface Sci. 2019, $42,121-136$

(40) Avanti Lipids Polar, I. Phase Transition Temperatures for Glycerophospholipids I Avanti Polar Lipids. 2018.

(41) Carpentier, L.; Bée, M.; Giroud-Godquin, A. M.; Maldivi, P.; Marchon, J. C. Alkyl Chain Motions in Columnar Mesophases. Mol. Phys. 1989, 68, 1367-1378.

(42) Blicher, A.; Wodzinska, K.; Fidorra, M.; Winterhalter, M.; Heimburg, T. The Temperature Dependence of Lipid Membrane Permeability, Its Quantized Nature, and the Influence of Anesthetics. Biophys. J. 2009, 96, 4581-4591.

(43) Chang, H. H.; Dea, P. K. Insights into the Dynamics of DMSO in Phosphatidylcholine Bilayers. Biophys. Chem. 2001, 94, 33-40.

(44) Cheng, C. Y.; Song, J.; Pas, J.; Meijer, L. H. H.; Han, S. DMSO Induces Dehydration near Lipid Membrane Surfaces. Biophys. J. 2015, 109, 330-339.

(45) Tristram-Nagle, S.; Moore, T.; Petrache, H. I.; Nagle, J. F. DMSO Produces a New Subgel Phase in DPPC: DSC and X-Ray Diffraction Study. Biochim. Biophys Acta - Biomembr. 1998, 1369, 1933.

(46) Kiselev, M. A.; Lesieur, P.; Kisselev, A. M.; GrabielleMadelmond, C.; Ollivon, M. DMSO-Induced Dehydration of DPPC Membranes Studied by X-Ray Diffraction, Small-Angle Neutron Scattering, and Calorimetry. J. Alloys Compd. 1999, 286, 195-202.

(47) Westh, P. Preferential Interaction of Dimethyl Sulfoxide and Phosphatidyl Choline Membranes. Biochim. Biophys. Acta - Biomembr. 2004, 1664, 217-223.

(48) Notman, R.; Noro, M.; O’Malley, B.; Anwar, J. Molecular Basis for Dimethylsulfoxide (DMSO) Action on Lipid Membranes. J. Am. Chem. Soc. 2006, 128, 13982-13983.

(49) de Ménorval, M. A.; Mir, L. M.; Fernández, M. L.; Reigada, R. Effects of Dimethyl Sulfoxide in Cholesterol-Containing Lipid Membranes: A Comparative Study of Experiments in Silico and with Cells. PLoS One 2012, 7, No. e41733.

(50) Schrader, A. M.; Cheng, C.-Y.; Israelachvili, J. N.; Han, S. Communication: Contrasting Effects of Glycerol and DMSO on Lipid Membrane Surface Hydration Dynamics and Forces. J. Chem. Phys. 2016, 145, No. 041101.

(51) Gironi, B.; Paolantoni, M.; Morresi, A.; Foggi, P.; Sassi, P. Influence of Dimethyl Sulfoxide on the Low-Temperature Behavior of Cholesterol-Loaded Palmitoyl-Oleyl-Phosphatidylcholine Membranes. J. Phys. Chem. B 2018, 122, 6396-6402.

(52) Doxastakis, M.; García Sakai, V.; Ohtake, S.; Maranas, J. K.; De Pablo, J. J. A Molecular View of Melting in Anhydrous Phospholipidic Membranes. Biophys. J. 2007, 92, 147-161.

(53) Sharma, V. K.; Mitra, S.; Verma, G.; Hassan, P. A.; Sakai, V. G.; Mukhopadhyay, R. Internal Dynamics in SDS Micelles: Neutron Scattering Study. J. Phys. Chem. B 2010, 114, 17049-17056.

(54) Nanda, H.; García Sakai, V.; Khodadadi, S.; Tyagi, M. S.; Schwalbach, E. J.; Curtis, J. E. Relaxation Dynamics of Saturated and Unsaturated Oriented Lipid Bilayers. Soft Matter 2018, 14, 61196127.

(55) Small, D. M. Lateral Chain Packing in Lipids and Membranes. J. Lipid Res. 1984, 25, 1490-1500.

(56) Grage, S. L.; Afonin, S.; Kara, S.; Buth, G.; Ulrich, A. S. Membrane Thinning and Thickening Induced by Membrane-Active Amphipathic Peptides. Front. Cell Dev. Biol. 2016, 4, 65. (JUN)

(57) Stadler, A. M.; Van Eijck, L.; Demmel, F.; Artmann, G. Macromolecular Dynamics in Red Blood Cells Investigated Using Neutron Spectroscopy. J. R. Soc., Interface 2011, 8, 590-600. 
(58) Chudley, C. T.; Elliott, R. J. Neutron Scattering from a Liquid on a Jump Diffusion Model. Proc. Phys. Soc. 1961, 77, 353-361. 